\title{
Efeito fungicida dos óleos essenciais de Schinus molle L. e Schinus terebinthifolius Raddi, Anacardiaceae, do Rio Grande do Sul
}

\author{
Ana C. Atti dos Santos, ${ }^{* 1,2}$ Marcelo Rossato, ${ }^{1,3}$ Luciana Atti Serafini, ${ }^{1,2}$ Marina Bueno, ${ }^{1}$ \\ Liziane B. Crippa, ${ }^{1}$ Valdirene C. Sartori, ${ }^{1}$ Eduardo Dellacassa, ${ }^{4}$ Patrick Moyna ${ }^{5}$ \\ ${ }^{1}$ Instituto de Biotecnologia, Universidade de Caxias do Sul, Rua Francisco Getúlio Vargas, 1130, \\ CEP 95001-970, Caxias do Sul-RS, Brazil \\ ${ }^{2}$ Departamento de Física e Química, Universidade de Caxias do Sul, Rua Francisco Getúlio Vargas, 1130, \\ CEP 95001-970, Caxias do Sul-RS, Brazil \\ ${ }^{3}$ Departamento de Ciências Biomédicas, Universidade de Caxias do Sul, Rua Francisco Getúlio Vargas, 1130, \\ CEP 95001-970, Caxias do Sul-RS, Brazil \\ ${ }^{4}$ Cátedra de Farmacognosia, Facultad de Quimica, UR, 11800, Montevideo, Uruguay \\ ${ }^{5}$ DEPTEQ, Facultad de Quimica, UR, 11800, Montevideo, Uruguay.
}

\begin{abstract}
RESUMO: Uma das alternativas para reduzir o uso de agrotóxicos pode ser a utilização de óleos essenciais para o controle de doenças e pragas que afetam a produção agrícola. O objetivo do presente estudo foi verificar o efeito fungicida dos óleos essenciais de Schinus molle L. e Schinus terebinthifolius Raddi, espécies nativas amplamente distribuídas na região. Os óleos foram extraídos por hidrodestilação e analisados por cromatografia gasosa. Os fungos fitopatógenos Alternaria spp., Botrytis spp., Colletotrichum spp. e Fusarium spp., foram isolados a partir de gérberas e rosas com sintomas de doença e os ensaios de atividade antifúngica foram realizados através da técnica de difusão em disco e os dados analisados pelo teste de Tukey e Duncan a 5\%. Em geral o óleo essencial de $S$. molle foi efetivo para os quatro fungos testados, nas quatro diluições utilizadas e para todos os tempos avaliados e o óleo essencial de $S$. terebinthifolius apresenta efeito fungicida mais pronunciado contra Botrytis spp., a partir de testes in vitro.
\end{abstract}

Unitermos: óleos essenciais, Schinus molle., Schinus terebinthifolius, efeito fungicida, $\alpha$-pineno, sabineno.

\begin{abstract}
Antifungal effect of Schinus molle L.,Anacardiaceae, and Schinusterebinthifolius Raddi, Anacardiaceae, essential oils of Rio Grande do Sul”. One of the alternatives to reduce the use of pesticides may be the use of essential oils to control diseases and pests that affect agricultural production. The purpose of this study was to evaluate the fungicide effect of essential oils of Schinus molle L. and Schinus terebinthifolius Raddi, native species widely distributed in the region. The oils were extracted by hydro distillation and analyzed by gas chromatography. The phytopathogenic fungi Alternaria spp., Botrytis spp., Colletotrichum spp. and Fusarium spp., were isolated from "gerberas" and roses with symptoms of disease and antifungal activity of the tests were carried out by the technique of spreading disk. The data was analyzed by Tukey and Duncan tests to $5 \%$. In general, the essential oil of $S$. molle was effective for the four fungi tested, for the four dilutions used and for all the times evaluated. The essential oil of S. terebinthifolius presents more pronounced fungicide effect against Botrytis spp., from in vitro tests.
\end{abstract}

Keywords: essential oils, Schinus molle., Schinus terebinthifolius, antifungal effect, $\alpha$-pinene, sabinene.

\section{INTRODUÇÃO}

Schinus terebinthifolius Raddi (aroeira-vermelha) e Schinus molle L. (aroeira-periquita) são espécies nativas da América do Sul, pertencentes à família Anacardiaceae. No Brasil, ocorrem principalmente de Pernambuco ao Rio Grande do Sul em diversos tipos de formações vegetais (Carvalho, 1994). Devido à grande dispersão das espécies, apresentam diversas aplicações, principalmente pela madeira, na extração de taninos, em paisagismo (Allardice et al., 1999; Guerra et al., 2000) e como medicinais. S. terebinthifolius é uma das plantas mais conhecidas popularmente no tratamento de inflamações uterinas (Amorin \& Santos, 2003) e na cicatrização de feridas e úlceras (Bacchi, 1986; Martínez et al., 1996) e tem sido comprovada cientificamente sua ação 
antimicrobiana (Siddiqui et al., 1995; Guerra et al., 2000) e antioxidante (Velázquez et al., 2003). S. molle possui propriedade antiespasmódica, anti-reumática, emenagoga, antiinflamatória e cicatrizante (Piva, 2002).

Além destas aplicações, ambas as espécies produzem, entre outros compostos, flavonóides, taninos e óleos essenciais (Lawrence, 1984; Queires \& Rodrigues, 1998). De todos os metabólitos secundários sintetizados pelos vegetais, os alcalóides e os óleos essenciais formam o grupo de compostos com maior número de substâncias biologicamente ativas (Di Stasi, 1996). Os óleos essenciais, em especial, atuam como inibidores da germinação, na proteção contra predadores, na atração de polinizadores, na proteção contra perda de água e aumento da temperatura (Bruneton, 1991; Simões \& Spitzer, 2000).

Trabalhos desenvolvidos com extratos brutos ou óleos essenciais, obtidos a partir de plantas medicinais têm indicado seu potencial no controle de fitopatógenos (Cunico et al., 1999), agentes causadores de doenças em vegetais que acarretam perdas significativas na produção, destruição de grãos durante a estocagem, diminuição do valor nutritivo e, algumas vezes, produção de micotoxinas prejudiciais ao homem e aos animais (Velluti et al., 2004). Segundo Bhavanani \& Ballow (1992) cerca de 60\% dos óleos essenciais possuem atividades antifúngicas e 35\% exibem propriedades antibacterianas.

Existem relatos sobre a atividade antifúngica (Dikshit et al., 1986), antimicrobiana (Gundidza, 1993), inseticida (Chantraine et al., 1998) e repelente (Wimalatne et al., 1996) de óleos essenciais de S. molle, assim como relatos da atividade antimicrobiana (Siddiqui et al., 1995) e fungistática (Siddiqui et al., 1996) de óleos essenciais de S. terebinthifolius.

Considerando estes fatores, o presente trabalho teve por objetivo avaliar o efeito fungicida dos óleos essenciais de S. molle e S. terebinthifolius do Rio Grande do Sul sobre o crescimento de Alternaria spp., Botrytis spp., Colletotrichum spp. e Fusarium spp., que causam perdas significativas na produção agrícola, proporcionando uma alternativa à utilização indiscriminada de agrotóxicos, e por sua vez reduzindo o custo de produção para o agricultor.

\section{MATERIAL E MÉTODOS}

\section{Óleos essenciais}

Para este estudo foi realizado um mix de óleos essenciais dos acessos 226, 232, 243, 265, 307, 310, 319, 324 e 331 de Schinus molle L. e um mix de óleos essenciais de quatro exemplares do acesso 102 de Schinus terebinthifolius Raddi. Plantas dos acessos foram catalogadas e registradas no Herbário da Universidade de Caxias do Sul - HUCS (Tabela 1).

Os óleos essenciais foram obtidos por hidrodestilação em Clevenger durante 1 h (Farmacopéia Brasileira, 1988). Foram analisadas as folhas desidratadas em secador de ervas, a $36{ }^{\circ} \mathrm{C}$, ventilação forçada de ar e controle de umidade.

\section{Análises cromatográficas}

A identificação dos componentes químicos foi feita por cromatografia gasosa (CG) e cromatografia gasosa acoplada a detector seletivo de massas (CG/MS). As análises em CG foram realizadas num cromatógrafo Hewlett Packard 6890, equipado com um processador de dados HP-Chemstation. As análises em coluna polar foram realizadas em coluna HP-Innowax (30 m x $320 \mu \mathrm{m}$ i.d.) $0,50 \mu \mathrm{m}$ de espessura de filme (Hewlett Packard, USA), com a seguinte programação de temperatura: $40{ }^{\circ} \mathrm{C}(8$ min) para $180{ }^{\circ} \mathrm{C} \mathrm{a} 3^{\circ} \mathrm{C} / \mathrm{min}, 180-230^{\circ} \mathrm{C}$ a $20^{\circ} \mathrm{C} / \mathrm{min}, 230$ ${ }^{\circ} \mathrm{C}$ (20 min); temperatura de injetor $250^{\circ} \mathrm{C}$; razão de split 1:50, temperatura do detector FID $250^{\circ} \mathrm{C}$; gás de arraste $\mathrm{H}_{2}$ (34 Kpa), volume injetado $1 \mu \mathrm{L}$ diluído em hexano $(1: 10)$.

As análises em GC/MS foram realizadas num cromatógrafo gasoso acoplado a detector seletivo de massas Hewlett Packard 6890/MSD5973, equipado com

Tabela 1. Acessos coletados em diferentes regiões do Rio Grande do Sul.

\begin{tabular}{|c|c|c|c|c|c|c|}
\hline \multirow{2}{*}{ Acesso } & \multirow{2}{*}{ Espécie } & \multirow{2}{*}{ Local } & \multicolumn{2}{|c|}{ Localização geográfica } & \multirow{2}{*}{$\begin{array}{l}\text { Número de } \\
\text { herbário }\end{array}$} & \multirow{2}{*}{ Determinador } \\
\hline & & & $\mathrm{S}$ & W & & \\
\hline 226 & S. molle & São Lourenço & $31^{\circ} 15^{\prime} 0,5,7^{\prime \prime}$ & $51^{\circ} 59^{\prime} 49,1^{\prime \prime}$ & 23987 & Stephen \& Smith \\
\hline 232 & S. molle & Cristal & $30^{\circ} 55^{\prime} 03,1 ”$ & $52^{\circ} 07^{\prime} 12,9^{\prime \prime}$ & 24441 & Stephen \& Smith \\
\hline 243 & S. molle & São Lourenço 2 & $31^{\circ} 12^{\prime} 31.3 ”$ & $52^{\circ} 01^{\prime} 05.1^{\prime \prime}$ & 24690 & Stephen \& Smith \\
\hline 265 & S. molle & Erechim & $27^{\circ} 38^{\prime} 02,7^{\prime}$ & $52^{\circ} 15^{\prime} 39,5^{\prime \prime}$ & 24697 & Stephen \& Smith \\
\hline 307 & S. molle & Pinheiro Machado & $31^{\circ} 36^{\prime} 18^{\prime \prime}$ & $53^{\circ} 18^{\prime} 50,1^{\prime \prime}$ & 26712 & Stephen \& Smith \\
\hline 310 & S. molle & Dom Pedrito & $31^{\circ} 02^{\prime} 04,8^{\prime \prime}$ & $54^{\circ} 33^{\prime} 56,7^{\prime \prime}$ & 27185 & M. Rossato \\
\hline 319 & S. molle & Quarai & $30^{\circ} 24^{\prime} 06,1^{\prime \prime}$ & $56^{\circ} 11^{\prime} 06,1^{\prime \prime}$ & 27187 & M. Rossato \\
\hline 324 & S. molle & São Borja & 2843’15,9” & $56^{\circ} 00^{\prime} 36,8^{\prime \prime}$ & 27188 & M. Rossato \\
\hline 331 & S. molle & São Miguel & $28^{\circ} 31^{\prime} 00^{\prime \prime}$ & $54^{\circ} 32^{\prime} 43,3^{\prime \prime}$ & 27189 & M. Rossato \\
\hline 102 & S. terebinthifolius & Caxias do Sul & $29^{\circ} 09^{\prime} 78,0^{\prime \prime}$ & $51^{\circ} 08 ' 65,9^{\prime \prime}$ & 29186 & M. Rossato \\
\hline
\end{tabular}


software HP Chemstation e espectroteca Wiley 275. As análises foram realizadas em coluna polar HP-Innowax (30 $\mathrm{m} \times 250 \mu \mathrm{m})$ 0,50 $\mu \mathrm{m}$ espessura de filme (Hewlett Packard, USA). O programa de temperatura utilizado foi o mesmo usado na análise em CG, interface $280{ }^{\circ} \mathrm{C}$; razão de split 1:100; gás de arraste He (56 Kpa); energia de ionização $70 \mathrm{eV}$; intervalo de aquisição de massas 40-350; solvent cut 3,5 min, volume injetado $0,4 \mu \mathrm{L}$ diluído em hexano (1:10).

\section{Ensaios da atividade antifúngica}

Foram testadas diluições de 25, 50, 75 e 100\% de óleo essencial em Tween 80 sobre os fungos Alternaria spp., Botrytis spp., Colletotrichum spp. e Fusarium spp., isolados a partir de gérberas e rosas com sintoma de doença. Cem microlitros de cada diluição foram espalhadas sobre a superfície do meio BDA em placas de Petri. Placas contendo somente BDA serviram como testemunha. Quatro discos de $3 \mathrm{~mm}$ de diâmetro de ágar, colonizados pelo fungo, foram transferidos para a placa contendo o óleo essencial, e em seguida incubadas à $28^{\circ} \mathrm{C}$ pelo período de até $72 \mathrm{~h}$, em duas repetições. A avaliação do efeito fungicida do óleo foi feito nos tempos de 24, 48 e 72 h após o repique, medindo-se o diâmetro da colônia e os dados analisados pelo teste de Tukey a 5\%.

A verificação da diluição mínima para inibir o crescimento de halo dos fungos avaliados foi realizada com diluições de 10, 15 e 20\%, nas mesmas condições descritas anteriormente. Os dados foram analisados pelo teste de Duncan a 5\%.

\section{RESULTADOS E DISCUSSÃO}

Os resultados mostraram que diluições inferiores a 50\% de óleo essencial de S. terebinthifolius não inibem o crescimento de Fusarium spp. Para os outros fungos testados, uma diluição de $25 \%$ já inibe o diâmetro de halo, para todos os tempos avaliados, quando comparados aos controles (Tabela 2).

Nesta mesma tabela pode-se observar que para Fusarium spp. e Alternaria spp., o crescimento do fungo em 72 h difere estatisticamente dos outros tempos, tanto para o controle, quanto para as diferentes diluições testadas.

O óleo essencial de S. terebinthifolius foi mais

Tabela 2. Inibição do tamanho das colônias de fungos fitopatógenos após tratamento dos fungos com diferentes concentrações de óleo essencial de Schinus terebinthifolius.

\begin{tabular}{|c|c|c|c|c|c|c|c|c|c|c|c|}
\hline \multirow[t]{2}{*}{ Fungos } & \multirow{2}{*}{$\begin{array}{l}\text { Conc. } \\
(\%)\end{array}$} & \multirow[t]{2}{*}{$\mathrm{n}$} & \multicolumn{3}{|c|}{ Tempo } & \multirow[t]{2}{*}{ Fungos } & \multirow{2}{*}{$\begin{array}{c}\text { Conc. } \\
(\%)\end{array}$} & \multirow[t]{2}{*}{$\mathrm{n}$} & \multicolumn{3}{|c|}{ Tempo } \\
\hline & & & $24 \mathrm{~h}$ & $48 \mathrm{~h}$ & $72 \mathrm{~h}$ & & & & $24 \mathrm{~h}$ & $48 \mathrm{~h}$ & $72 \mathrm{~h}$ \\
\hline \multirow{5}{*}{ Fusarium spp. } & 0 & 12 & 7,04 Ca & $24,02 \mathrm{Ba}$ & 36,61 Aa & \multirow{5}{*}{ Alternaria spp. } & 0 & 12 & 9,98 Ca & $21,16 \mathrm{Ba}$ & $29,06 \mathrm{Aa}$ \\
\hline & 25 & 24 & $5,28 \mathrm{Ca}$ & $17,85 \mathrm{Ba}$ & 26,94 Аа & & 25 & 24 & 4,89 Cb & $10,46 \mathrm{Bb}$ & $15,93 \mathrm{Ab}$ \\
\hline & 50 & 24 & $0 \mathrm{Cb}$ & $6,4 \mathrm{Bb}$ & $10,01 \mathrm{Ab}$ & &, 50 & 24 & $0 \mathrm{Cb}$ & $9,03 \mathrm{Bb}$ & $13,2 \mathrm{Ab}$ \\
\hline & 75 & 24 & $0 \mathrm{Bb}$ & $0 \mathrm{Bc}$ & 6,33 Ab & & 75 & 24 & $0 \mathrm{Cb}$ & $7,12 \mathrm{Bb}$ & $11,3 \mathrm{Ab}$ \\
\hline & 100 & 24 & $0 \mathrm{Bb}$ & $0 \mathrm{Bc}$ & 5,38 Ab & & 100 & 24 & $0 \mathrm{Cb}$ & $6,71 \mathrm{Bb}$ & $10,45 \mathrm{Ab}$ \\
\hline \multirow{5}{*}{$\begin{array}{l}\text { Colletotrichum } \\
\text { spp. }\end{array}$} & 0 & 12 & 6,45 Ca & $20,98 \mathrm{Ba}$ & $27,87 \mathrm{Aa}$ & \multirow{5}{*}{ Botrytis spp. } & 0 & 12 & 5,14 Ca & $15,06 \mathrm{Ba}$ & 22,9 Аа \\
\hline & 25 & 24 & $0 \mathrm{Bb}$ & $10,32 \mathrm{Ab}$ & $14,99 \mathrm{Ab}$ & & 25 & 24 & $0 \mathrm{Ab}$ & $0 \mathrm{Ab}$ & $0 \mathrm{Ab}$ \\
\hline & 50 & 24 & $0 \mathrm{Bb}$ & 9,76 Ab & $13,44 \mathrm{Ab}$ & & 50 & 24 & $0 \mathrm{Ab}$ & $0 \mathrm{Ab}$ & $0 \mathrm{Ab}$ \\
\hline & 75 & 24 & $0 \mathrm{Bb}$ & 9,75 Ab & $14,55 \mathrm{Ab}$ & & 75 & 24 & $0 \mathrm{Ab}$ & $0 \mathrm{Ab}$ & $0 \mathrm{Ab}$ \\
\hline & 100 & 24 & $0 \mathrm{Bb}$ & 8,97 Ab & $14,15 \mathrm{Ab}$ & & 100 & 24 & $0 \mathrm{Ab}$ & $0 \mathrm{Ab}$ & $0 \mathrm{Ab}$ \\
\hline
\end{tabular}

Teste de Tukey a 5\% de probabilidade. Maiúsculas entre coluna e minúsculas entre linhas.

Tabela 3. Diâmetro de halo médio entre as placas de controle a as contendo óleo essencial de Schinus terebinthifolius.

\begin{tabular}{lcc}
\hline \multicolumn{1}{c}{ Fungos } & $\mathrm{n}$ & diâmetro de halo (mm) \\
\hline Fusarium spp. & 96 & $6,56 \mathrm{c}$ \\
Colletotrichum spp. & 96 & $8,00 \mathrm{c}$ \\
Alternaria spp. & 96 & $7,42 \mathrm{c}$ \\
Botrytis spp. & 96 & $0,29 \mathrm{~d}$ \\
Controle Fusarium spp. & 12 & $22,55 \mathrm{a}$ \\
Controle Colletotrichum spp. & 12 & $18,83 \mathrm{ab}$ \\
Controle Alternaria spp. & 12 & $20,06 \mathrm{ab}$ \\
Controle Botrytis spp. & 12 & $14,36 \mathrm{~b}$ \\
\hline
\end{tabular}

Teste de Tukey a 5\% de probabilidade 
efetivo para Botrytis spp., onde observa-se a inibição do crescimento do halo, em todas as diluições testadas, para todos os tempos avaliados (Tabela 2). Este efeito pode estar relacionado ao diâmetro de halo médio deste fungo, que apresentou-se inferior aos demais fungos (Tabela 3).

Para S. molle, observou-se que em Fusarium spp. e Botrytis spp., diluições acima de 25\% de óleo essencial inibem o crescimento dos fungos. Em Colletotrichum spp. e Alternaria spp., é necessário 50\% de óleo essencial, pois há crescimento do fungo com aplicação de $25 \%$ de óleo a partir de 48 h de avaliação. Para estes dois fungos, o diâmetro de halo entre o controle e utilizando a diluição de $25 \%$ diferem estatisticamente de $72 \mathrm{~h}$, para 40 e $24 \mathrm{~h}$ (Tabela 4).

Tabela 4. Inibição do tamanho das colônias de fungos fitopatógenos após tratamento dos fungos com diferentes concentrações de óleo essencial de Schinus molle.

\begin{tabular}{|c|c|c|c|c|c|c|c|c|c|c|c|}
\hline \multirow[t]{2}{*}{ Fungos } & \multirow{2}{*}{$\begin{array}{l}\text { Conc. } \\
(\%)\end{array}$} & \multirow[t]{2}{*}{$\mathrm{n}$} & \multicolumn{3}{|c|}{ Tempo } & \multirow[t]{2}{*}{ Fungos } & \multirow{2}{*}{$\begin{array}{l}\text { Conc. } \\
(\%)\end{array}$} & \multirow[t]{2}{*}{$\mathrm{n}$} & \multicolumn{3}{|c|}{ Tempo } \\
\hline & & & $24 \mathrm{~h}$ & $48 \mathrm{~h}$ & $72 \mathrm{~h}$ & & & & $24 \mathrm{~h}$ & $48 \mathrm{~h}$ & $72 \mathrm{~h}$ \\
\hline \multirow{5}{*}{ Fusarium spp. } & 0 & 12 & $9,32 \mathrm{Ca}$ & $26,09 \mathrm{Ba}$ & 44,26 Аа & \multirow{5}{*}{ Alternaria spp. } & 0 & 12 & $8,44 \mathrm{Ca}$ & $21,49 \mathrm{Ba}$ & $34,42 \mathrm{Aa}$ \\
\hline & 25 & 24 & $0 \mathrm{Ab}$ & $0 \mathrm{Ab}$ & $0 \mathrm{Ab}$ & & 25 & 24 & $0 \mathrm{Cb}$ & 4,51 Bb & $8,15 \mathrm{Ab}$ \\
\hline & 50 & 24 & $0 \mathrm{Ab}$ & $0 \mathrm{Ab}$ & $0 \mathrm{Ab}$ & & 50 & 24 & $0 \mathrm{Ab}$ & $0 \mathrm{Ac}$ & 0 Ac \\
\hline & 75 & 24 & $0 \mathrm{Ab}$ & $0 \mathrm{Ab}$ & $0 \mathrm{Ab}$ & & 75 & 24 & $0 \mathrm{Ab}$ & 0 Ac & 0 Ac \\
\hline & 100 & 24 & $0 \mathrm{Ab}$ & $0 \mathrm{Ab}$ & $0 \mathrm{Ab}$ & & 100 & 24 & $0 \mathrm{Ab}$ & 0 Ac & 0 Ac \\
\hline \multirow{5}{*}{$\begin{array}{l}\text { Colletotrichum } \\
\text { spp. }\end{array}$} & 0 & 12 & 7,19 Ca & $18,38 \mathrm{Ba}$ & 28,46 Аа & \multirow{5}{*}{ Botrytis spp. } & 0 & 12 & 4,89 Ca & 8,75 Ba & $13,10 \mathrm{Aa}$ \\
\hline & 25 & 24 & $0 \mathrm{Ab}$ & 5,54 Bb & $9,46 \mathrm{Ab}$ & & 25 & 24 & $0 \mathrm{Ab}$ & $0 \mathrm{Ab}$ & $0 \mathrm{Ab}$ \\
\hline & 50 & 24 & $0 \mathrm{Ab}$ & $0 \mathrm{Ac}$ & 0 Ac & & 50 & 24 & $0 \mathrm{Ab}$ & $0 \mathrm{Ab}$ & $0 \mathrm{Ab}$ \\
\hline & 75 & 24 & $0 \mathrm{Ab}$ & $0 \mathrm{Ac}$ & 0 Ac & & 75 & 24 & $0 \mathrm{Ab}$ & $0 \mathrm{Ab}$ & $0 \mathrm{Ab}$ \\
\hline & 100 & 24 & $0 \mathrm{Ab}$ & $0 \mathrm{Ac}$ & 0 Ac & & 100 & 24 & $0 \mathrm{Ab}$ & $0 \mathrm{Ab}$ & $0 \mathrm{Ab}$ \\
\hline
\end{tabular}

Teste de Tukey a 5\% de probabilidade. Maiúsculas entre coluna e minúsculas entre linhas.

Em geral o óleo essencial de $S$. molle foi efetivo para os quatro fungos testados, nas quatro diluições utilizadas e para todos os tempos avaliados (Tabela 5).

Os resultados obtidos com a utilização de diluições inferiores a $25 \%$ mostram que os fungos comportam-se diferentemente tanto em relação ao óleo utilizado como em relação à diluição letal ou à diluição necessária para inibir o crescimento do fungo (Tabela 6). O óleo essencial de S. molle é letal para Botrytis spp. e Fusarium spp. em diluições inferiores a 10\%, porém para Alternaria spp. e Colletotrichum spp. são necessárias diluições superiores a 10 e $25 \%$, respectivamente.

Para S. terebinthifolius, observou-se que o óleo essencial não apresenta efeito fungicida contra Colletotrichum spp. e Fusarium spp., porém pode-se inibir o crescimento dos fungos utilizando diluições superiores a $10 \%$ para Colletotrichum spp. e inferiores a $10 \%$ para Fusarium spp. Alternaria spp. e Botrytis spp. mostraramse mais resistentes ao óleo de $S$. terebinthifolius, quando comparados aos outros fungos testados.

Quimicamente, o óleo essencial de $S$. terebinthifolius apresentou $\alpha$-pineno, sabineno e biciclogermacreno e o óleo essencial de $S$. molle apresentou $\alpha$-pineno, sabineno como compostos majoritários. $\alpha$-Pineno e sabineno possuem atividade bactericida comprovada por testes com a espécie Lithraea molleoides, conhecida popularmente como aroeira branca (Shimizy et al., 2006). O biciclogermacreno não é citado quanto à atividade bactericida, porém possui potencial larvicida comprovado a partir de testes com as larvas do mosquito Aedes aegypti (Santos et al., 2006).
Souza e colaboradores (2005) referenciam a maior ou menor atividade biológica dos óleos essenciais dependente de alguns constituintes químicos em especial (citral, pineno, cineol, cariofileno, elemeno, furanodieno, limoneno, eugenol e carvacrol), porém é importante comentar que devido à complexidade da composição química de um óleo essencial, torna-se difícil relacionar a atividade biológica com as substâncias presentes. Geralmente, a ação atribuída a um composto isolado pode não ser exata, devido a possíveis interações que podem ocorrer entre os compostos do óleo (Apel, 2001).

Apesar de não terem sidos realizados ensaios in vivo, os resultados obtidos com este estudo demonstram que o óleo essencial de S. molle apresenta efeito fungicida contra Alternaria spp., Fusarium spp., Collethotricum spp. e Botrytis spp. e o óleo essencial de S. terebinthifolius apresenta efeito fungicida mais pronunciado contra Botrytis spp., a partir de testes in vitro.

Tabela 5. Diâmetro de halo médio entre as placas de controle a as contendo óleo essencial de Schinus molle.

\begin{tabular}{lcc}
\hline \multicolumn{1}{c}{ Fungos } & $\mathrm{n}$ & diâmetro de halo (mm) \\
\hline Fusarium spp. & 96 & $0 \mathrm{~d}$ \\
Colletotrichum spp. & 96 & $1,25 \mathrm{~d}$ \\
Alternaria spp. & 96 & $1,06 \mathrm{~d}$ \\
Botrytis spp. & 96 & $0 \mathrm{~d}$ \\
Controle Fusarium spp. & 12 & $26,56 \mathrm{a}$ \\
Controle Colletotrichum spp. & 12 & $18,01 \mathrm{~b}$ \\
Controle Alternaria spp. & 12 & $24,15 \mathrm{~b}$ \\
Controle Botrytis spp. & 12 & $8,91 \mathrm{c}$ \\
\hline
\end{tabular}

Teste de Tukey a 5\% de probabilidade. 
Tabela 6. Atividade dos óleos essenciais de S. molle e S. terebinthifolius em diferentes espécies de fungos fitopatógenos.

\begin{tabular}{|c|c|c|c|}
\hline Óleo essencial & Espécie de fungo & Diluição para inibição & Diluição letal \\
\hline \multirow{4}{*}{ S. molle } & Alternaria spp. & $<10 \%$ & $>10 \%$ \\
\hline & Botrytis spp. & Efeito letal & $<10 \%$ \\
\hline & Colletotrichum spp. & $<20 \%$ & $>25 \%$ \\
\hline & Fusarium spp. & Efeito letal & $<10 \%$ \\
\hline \multirow{4}{*}{ S. terebinthifolius } & Alternaria spp. & $<10 \%$ & $>50 \%$ \\
\hline & Botrytis spp. & $<20 \%$ & $>25 \%$ \\
\hline & Colletotrichum spp. & $>10 \%$ & Não possui \\
\hline & Fusarium spp. & $<10 \%$ & Não possui \\
\hline
\end{tabular}

Teste de Duncan a 5\% de probabilidade, valores avaliados pelos intervalos de confiança.

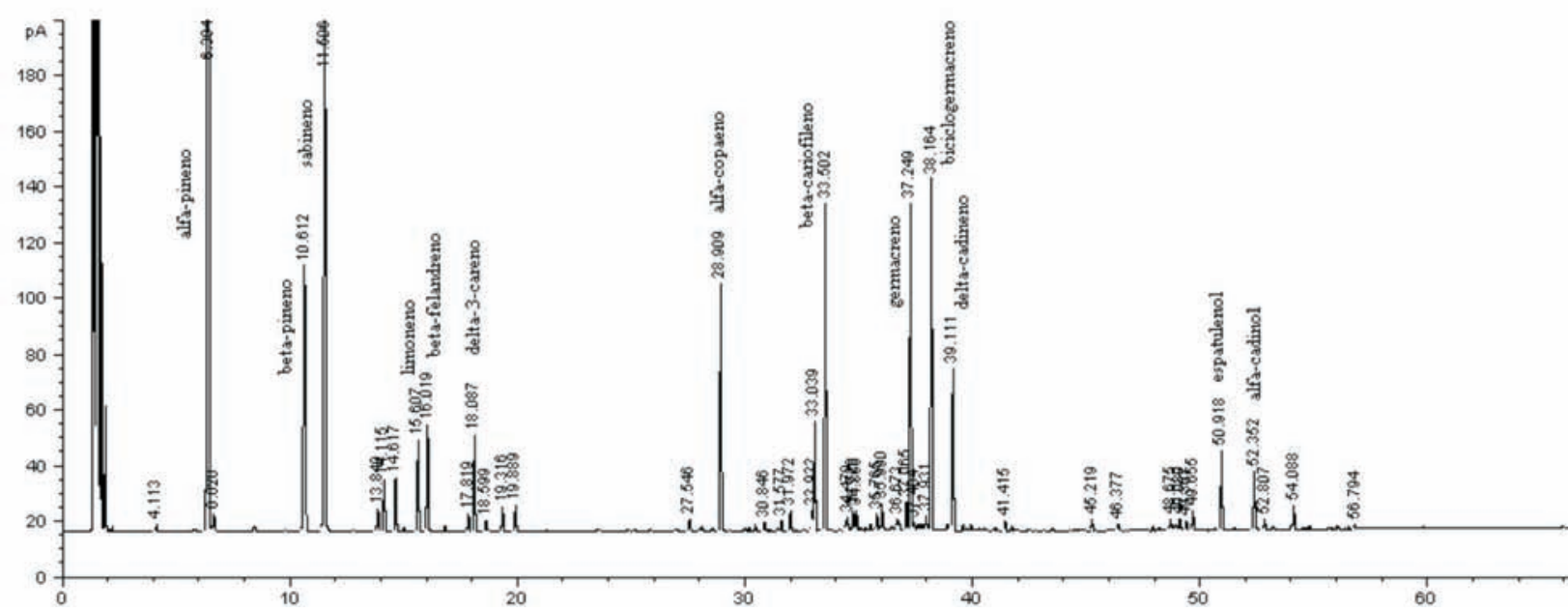

Figura 1. Perfil cromatográfico do mix do óleo essencial de Schinus terebinthifolius Raddi.

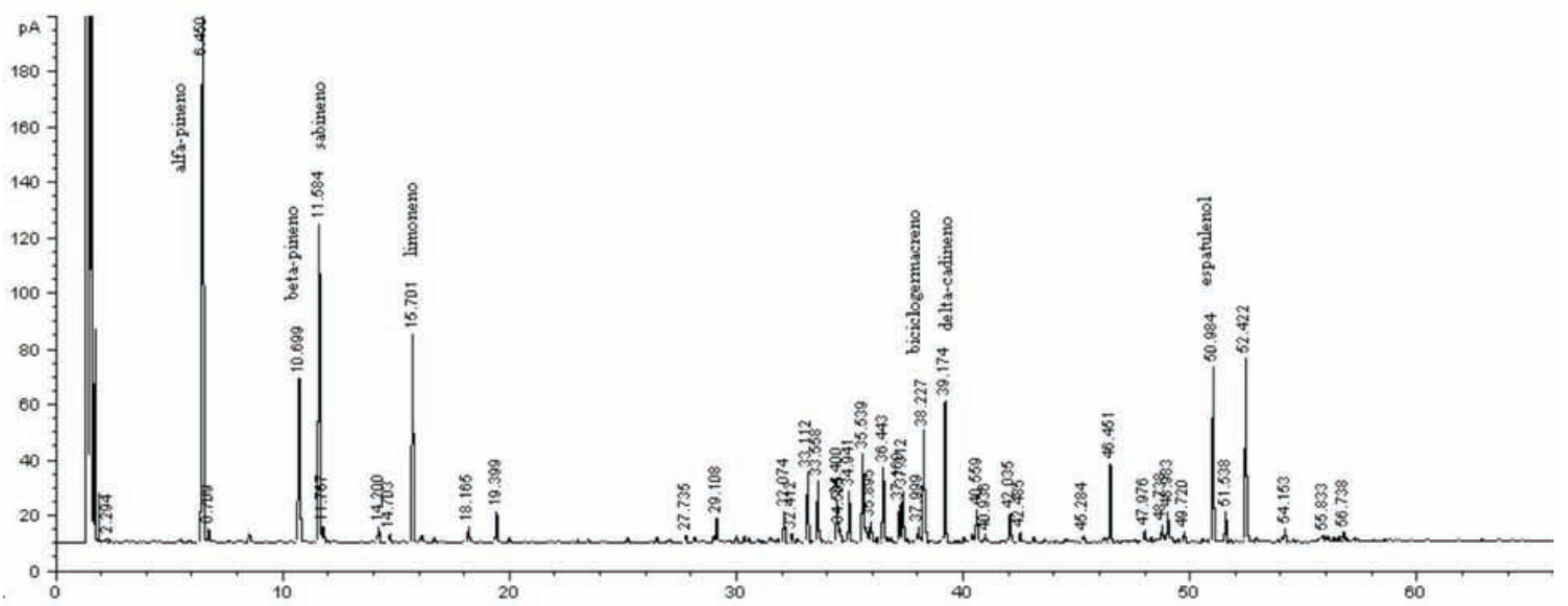

Figura 2. Perfil cromatográfico do mix do óleo essencial de Schinus molle L. 


\section{REFERÊNCIAS}

Apel MA 2001. Óleos voláteis de espécies da subtribo eugeniinae (Myrtaceae): composição química e atividades antimicrobiana e antiinflamatória. Porto Alegre, 256p. Tese de Doutorado, Ciências Farmacêuticas, Universidade Federal do Rio Grande do Sul.

Allardice P, Bone K, Hutchison F 1999. Segredos e virtudes das plantas medicinais. Rio de Janeiro: Editora Reader's Digest Brasil Ltda.

Amorin MMR, Santos LC 2003. Tratamento de vaginose bacteriana com gel vaginal de Aroeira (Schinus terebinthifolius Raddi): ensaio clínico randomizado. Rev Bras Ginec Obstetr 25: 95-102.

Bacchi EM 1986. Ação anti-úlcera e cicatrizante de algumas plantas brasileiras. Rev Bras Farmacogn 1: 93-100.

Bhavanani SM, Balow CH 1992. New agents for gram-positive bactéria. Curr Opin Microbiol 13: 528-534.

Bruneton J 1991. Elementos de fitoquímica y de farmacognosia. Zaragoza: Editorial Acribia.

Carvalho PER 1994. Espécies florestais brasileiras: recomendações silviculturais, potencialidades e uso da madeira. Brasília: EMBRAPA.

Chantraine JM, Laurent D, Ballivian C, Saavedra G, Ibanez R, Vilaseca A 1998. Insecticidal activity of essential oils on Aedes aegypti larvae. Phytother Res 12: 350-354.

Cunico MM, Miguel OG, Carvalho, JLS, Peitz C, Stamgarlin JR, Schwan-Estrada KRF, Cruz MES, Nozaki, MH 1999. Plantas medicinais e controle alternativo de fitopatógenos. Biotecnol Cien Desenvol 11: 16-21.

Dikshit A, Naqvi AA, Husain A 1986. Schinus molle: a new source of natural fungitoxicant. Appl Envoron Microbiol 51: $1085-1088$.

Di Stasi LC 1996. Plantas medicinais: arte e ciência. São Paulo: Editora UNESP.

Guerra MJM, Barreiro, ML, Rodriguez ZM, Rubaicaba Y 2000. Actividad antimicrobiana de um extracto fluido al $80 \%$ de Schinus terebinthifolius Raddi (copal). Rev Cub Plantas Med 5: 23-25.

Gundidza M 1993. Antimicrobial activity of essential oil from Schinus molle Linn. Central. Afr J Med 39: 231-234.

Lawrence B 1984. A discussion of Schinus molle and Schinus terebinthifolius. Perfumer \& Flavorist 9: 65-69.

Martínez MJ, Betancourt J, Alonso-Gonzalez N, Jauregui A 1996. Screening of some Cuban medicinal plants for antimicrobial activity. J Ethnopharmacol 52: 171-174.

Piva MG 2002. O caminho das plantas medicinais: estudo etnobotânico. Rio de Janeiro: Mondriam.

Queires LCS, Rodrigues LEA 1998. Quantificação das substâncias fenólicas totais em órgãos da aroeira Schinus terebinthifolius (Raddi). Braz Arch Biol Technol 41: 247 253.

Santos RP, Nunes EP, Nascimento RF 2006. Chemical composition and larvicidal activity of the essential oils of Cordialeucomalloides and Cordia curassavica from the Northeast of Brazil. J Braz Chem Soc 17: 1027-1030.
Siddiqui RR, Zafar U, Chaudhry SS, Ahmad H 1995. Antimicrobial activity of essential oils from Schinus terebinthifolius, Cypress sempervirens, Citrus limon, Ferula asafetida. Part I. Pak J Scient Ind Res 38: 358-361.

Siddiqui RR, Ahmad H, Shakoor CS, Ehteshamuddin AFM, Shireen S 1996. Antimicrobial activity of essential oils. Part II. Pak J Scient Ind Res 39: 43-47.

Shimizu MT, Bueno LJF, Rodrigues RFO 2006. Óleo essencial de Lithraea molleoides (Vell.): composição química e atividade antimicrobiana. Braz J Microbiol 37: 556-560.

Simões CMO, Spitzer V 2001. Óleos Voláteis. In: Simões CMO, Schenkel EP, Gosmann G, Mello JCP, Mentz LA, Petrovick PR Farmacognosia da Planta ao medicamento. Porto Alegre: Editora da Universidade Federal do Rio Grande do Sul, p. 397-426.

Souza EL, Lima EO, Freire KRL, Sousa CP 2005. Inibitory action of some essential oils and phytochemicals on the growth of moulds isolated from foods. Braz Arch Biol Technol 48: 245-250.

Velázquez E, Tournier HA, Mordujovich de Buschiazzo P, Saavedra G, Schinella GR 2003. Antioxidant activity of Paraguayan plant extracts. Fitoterapia 74: 91-97.

Velluti A, Sanchis V, Ramos AJ, Turon C, Marín S 2004. Impact of essential oils on growth rate, zearalenone and deoxynivalenol production by Fusarium graminearum under different temperature and water activity conditions in maize grain. $J$ Appl Microbiol 96: 716-724.

Wimalaratne PDC, Slessor KN, Borden JH, Chong LJ, Abate T 1996. Isolation and identification of house fly, Musca domestica L., repellents from pepper tree, Schinus molle L. J Chem Ecol 22: 49-59. 